Purdue University Purdue e-Pubs

2000

Noise Source Identification in a Rotary Compressor: A Multidisciplinary Synergetic Approach

\author{
H.J. Kim \\ Arvin Industries \\ Y.M. Cho \\ Seoul National University \\ R. Chou \\ United Technologies Carrier
}

Follow this and additional works at: https://docs.lib.purdue.edu/icec

Kim, H. J.; Cho, Y. M.; and Chou, R., "Noise Source Identification in a Rotary Compressor: A Multidisciplinary Synergetic Approach" (2000). International Compressor Engineering Conference. Paper 1437.

https://docs.lib.purdue.edu/icec/1437

This document has been made available through Purdue e-Pubs, a service of the Purdue University Libraries. Please contact epubs@purdue.edu for additional information.

Complete proceedings may be acquired in print and on CD-ROM directly from the Ray W. Herrick Laboratories at https://engineering.purdue.edu/ Herrick/Events/orderlit.html 


\title{
Noise Source Identification in a Rotary Compressor: A Multidisciplinary Synergetic Approach ${ }^{1}$
}

\author{
Han-Jun Kim ${ }^{1}$, Young Man $\mathrm{Cho}^{2}$ and Rudy Chou ${ }^{3}$ \\ ${ }^{1}$ Arvin Industries, Inc., $153113^{\text {th }}$ Street, Columbus, Indiana 47201 \\ ${ }^{2}$ School of Mechanical and Aerospace Engineering, Seoul National University, Seoul 151-742, Korea \\ ${ }^{3}$ United Technologies Carrier, Carrier Parkway, Syracuse, New York 13221
}

\begin{abstract}
The complex nature of noise/vibration generation and transmission in rotating machinery has made it indispensable to employ various techniques to identify the corresponding sources and paths. A theoretical approach via finite element analysis (FEA), an experimental approach via direct measurement, and a signal processing approach via feature extraction of the measured data have all made significant contributions. Yet, there has not been much inter-disciplinary effort among the three areas although their mutually complementary roles have long been recognized. In this paper, a synergetic approach is proposed to noise/vibration source and transmission path identification in order to take advantage of all the merits of the three different approaches. The proposed approach is discussed with a rotary compressor as a testbed. In the new approach, the experimentally measured sound intensity map is initially used to suggest the active radiation area, which guides us to instrument the rotary compressor at the suspected noise/vibration source positions. The collected data from the compressor is then processed to unravel the candidate source(s). Various signal processing techniques such as filtering, Hilbert transform, and fast Fourier transform (FFT) are used to enhance the desired features buried in the experimental data. Finally, modal analysis via FEA and direct measurement further help to pinpoint the true source(s) and simultaneously verifies the conjecture following the experimental data processing.
\end{abstract}

Keywords: intensity map, vibration-induced noise, timing mark, band-pass filtering, Hilbert transform, modal analysis

\section{INTRODUCTION}

It has long been recognized that the comfort level of any commercial product plays a role as a definite market discriminator. It is an irreversible trend that the importance of the comfort level is exceeding that of the functionality, as the living standard of human beings is ever improving. This is especially true for residential systems, e.g., air-conditioners, refrigerators, vacuum cleaners, etc. Among many factors determining the comfort level, acoustic noise and mechanical vibration are two nuisances that affect human perception most and, accordingly, have been studied intensively in Harris (1957), Croker (1975), Yerges (1978), Beranek (1971), Foreman (1990), Ewins (1984), and Allemang (1984).

The complex nature of noise/vibration generation and transmission in these residential systems naturally requires efforts from multiple disciplines for successful control of the comfort level. Modal analysis via finite element method (FEM) has offered tremendous insight into the qualitative understanding of noise/vibration generation and transmission without explicit instrumentation. On the other hand, the direct measurement of noise/vibration such as obtaining a sound intensity map has presented a guideline to the localization of noise/vibration sources. Signal processing techniques have provided another alternative by extracting relevant features from raw experimental data or simulation output that are not often self-revealing and by presenting these features in a more readily analyzable manner. Notwithstanding their individual success in attacking the problem

${ }^{1}$ This work has been supported by United Technologies Carrier, the Brain Korea 21 Project, Institute of Advanced Machinery and Design at Seoul National University.

Fifteenth International Compressor Engineering Conference at Purdue University, Weșt Lafayette, IN, USA - July 25-28, 2000 
of noise/vibration generation and transmission, the extent of multi-disciplinary efforts has been quite limited due to the significant gap between different disciplines. In this paper, a synergetic approach is proposed to noise/vibration source and transmission path identification by employing several techniques from multiple disciplines in order to take advantage of the merits of each technique at different stages of problem solving. A prima-facie case is made to demonstrate the role that the synergetic approach can play in the identification of noise/vibration sources and transmission paths. Despite its virtually unlimited realm of application, the proposed approach is discussed with a rotary compressor as a testbed for simplicity and clarity.

First, the frequency range of interest (typically narrow-band) is determined where the noise of the rotary compressor is actively radiating. Then, the sound intensity map around the compressor shows where the excessive sound radiation is physically emanating. The vibration is measured on the compressor shell where the sound intensity map indicates high radiation. The vibration signals are analyzed via various signal processing techniques to identify noise sources and transmission paths. A priori knowledge about the compressor running cycle is then used to locate the noise sources. Modal analysis via FEM and direct measurement of the cylinder pressure helps to pinpoint the noise source(s). Two noise generation mechanisms are identified in the rotary compressor via the proposed technique: over-pressurization in the cylinder chamber and structural resonance of the cylinder.

Section 2 shows how acoustic radiation is distributed in the rotary compressor, which leads to further instrumentation of vibration and pressure signals in the compressor. Section 3 describes the signal processing applications to extract the desired features from the measurements. In Section 4, the noise sources are finally determined by virtue of internal measurement and modal analysis.

\section{SOUND INTENSITY MAP ANAYSIS}

Identification of noise/vibration sources and transmission paths begins with defining the frequency range of interest, since in most cases the frequency content of discomforting noise/vibration is narrow-band rather than broad-band. In the rotary compressor under consideration, the $5 \mathrm{kHz}$ band is of our interest. Our goal is to localize this $5 \mathrm{kHz}$ band noise so that a remedy may be developed. The requirement for the experimental data collection naturally leads to an "outside-to-inside" approach, where experimental data are collected in the following order: 1) outside the compressor, 2) on the compressor shell, and 3) inside the compressor. For example, the internal instrumentation via an accelerometer(s) is much harder to achieve than the external instrumentation via sound intensity measurement using a microphone(s). In the "outside-to-inside" approach, the external instrumentation sheds light on the potential noise source(s), which alleviates the burden of detailed internal instrumentation. The alternative is to instrument a compressor in detail internally from the beginning.

The operating condition of the compressor is as follows: condensing temperature $130^{\circ} \mathrm{F}$, evaporating temperature $45^{\circ} \mathrm{F}$ and returned gas temperature $65^{\circ} \mathrm{F}$. The sound intensity map of the rotary compressor is measured with an array of microphones around the compressor. Fig. 1 gives an unwrapped view of the sound intensity map around the cylindrical surface of the compressor, where the intensity is measured in the $1 / 3$ octave band centered at $5 \mathrm{kHz}$. The microphone array is placed very close to the compressor shell so that the sound radiation in the direction normal to the compressor shell is measured. The intensity is represented in the gray scale, i.e., the lighter the color is, the stronger the sound intensity is. It is obvious in Fig. 1 that the excessive sound at the $5 \mathrm{kHz}$ band was mostly radiated from one location (location " $A$ "), which is close to the discharge port in the compressor. The intensity level at the location " $\mathrm{A}$ " region is about $10 \mathrm{~dB}$ higher than anywhere else on the compressor shell, which suggests measuring the vibration (or acceleration) at that location and examining the coherence between the vibration and the sound intensity.

Fig. 2 shows the schematic of the rotary compressor cylinder. The state of the rotary compressor during the compression cycle is represented by the crank angle throughout this paper. The crank angles are measured counterclockwise from the top of the page in Fig. 2. The vibration at the location " $\mathrm{A}$ " is measured with an accelerometer in the time domain and is shown in Fig. 3. The sampling rate is $16 \mathrm{kHz}$. The Fourier transform of 
the vibration signal at the location "A" indicates the existence of strong vibration peaks in the $5 \mathrm{kHz}$ band. Then, the coherence between the vibration and the sound intensity in the $5 \mathrm{kHz}$ band is measured in order to determine whether the $5 \mathrm{kHz}$ vibration is the cause of the $5 \mathrm{kHz}$ sound radiation. Such a coherence test can eliminate any possible existence of a nonlinear sound radiation mechanism that would nullify the subsequent analysis of the $5 \mathrm{kHz}$ vibration signal. It is always possible that the vibration at different frequency band can be shifted into the 5 $\mathrm{kHz}$ band when it generates the noise via a nonlinear mechanism. The measured coherence at $5 \mathrm{kHz}$ between the vibration and the sound radiation is 0.95 , which proves that the $5 \mathrm{kHz}$ noise is truly vibration-induced and that the radiation mechanism is linear. Once the origin of the noise in the $5 \mathrm{kHz}$ band is identified as the vibration at the location " $\mathrm{A}$ ", it is sufficient to analyze the vibration signal instead of the unwieldy sound intensity map, in order to perform the noise source and transmission path identification. In what follows, various signal processing techniques such as filtering, Hilbert transform, and fast Fourier transformare employed to analyze the vibration signals

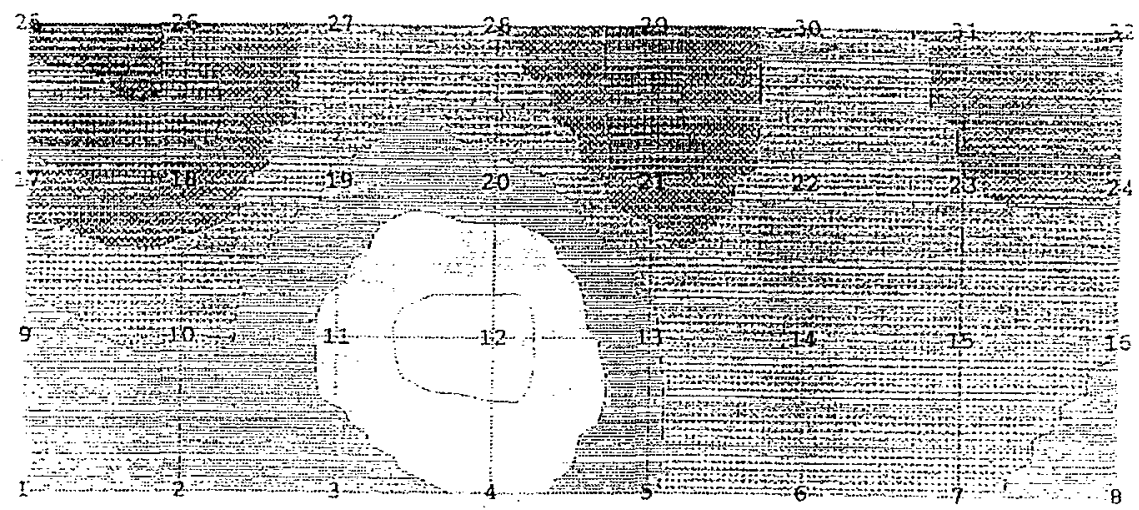

Fig. 1. Sound intensity map of the rotary compressor in the gray scale. The lighter the color is, the stronger the sound intensity is

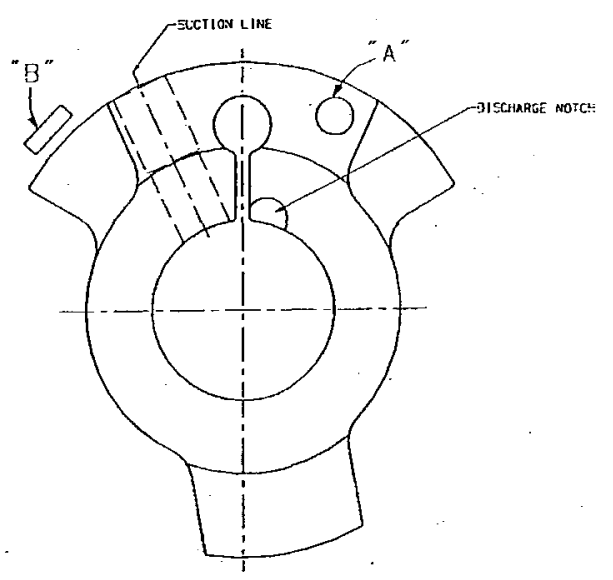

Fig. 2. Schematic diagram of the rotary compressor cylinder. The crank angle is measured counterclockwise from the top of the page.

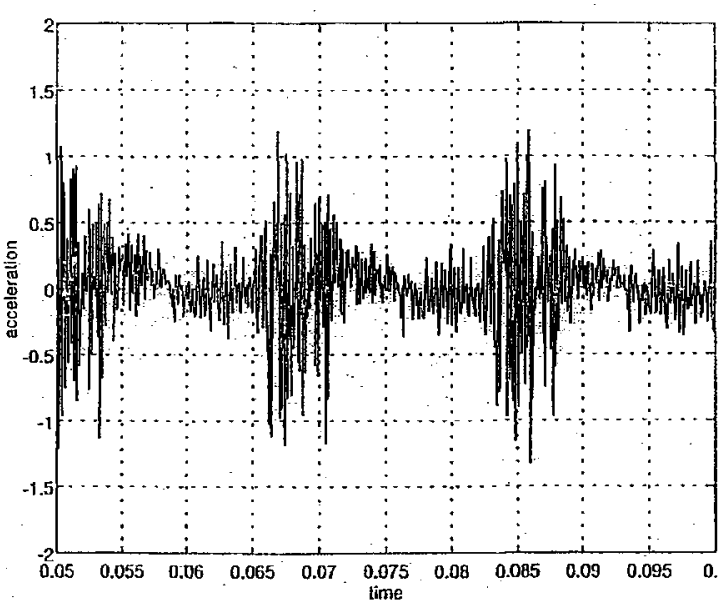

Fig. 3. Acceleration in the time domain at the location "A" 


\section{FEATURE EXTRACTION VIA SIGNAL PROCESSING}

In this section, various signal processing techniques are used to extract the necessary features for identifying noise sources in the $5 \mathrm{kHz}$ band. Our approach is to 1) locate the time instant when the maximum vibration occurs, 2) map the time instant into the crank angle, and 3) identify the possible vibration source(s) from the crank angle of the maximum vibration. In this respect, the signal processing techniques adopted in this section should not corrupt the $5 \mathrm{kHz}$ band component or change the timing (or phase) information of the signal. Throughout this section, it is worthwhile noting how such features are preserved.

\subsection{Band-Pass Filtering of Vibration Signals}

The time domain signal of vibration contains some unwanted information. A fourth order Butterworth filter, with pass band $4800-5200 \mathrm{~Hz}$, is designed to isolate the $5 \mathrm{kHz}$ component of the vibration signals. The MATLAB signal processing toolbox was used for the filter design (Mathworks, Inc., 1992). Since any causal filter inevitably introduces some phase delay into the filtered signal, special attention must be paid in order to preserve the phase information of the signal, which turns out to be crucial to noise source identification. Since a causal filter cannot preserve the phase, the concept of forward-backward filtering is adopted. Through the forward-backward filtering, the phase distortion is corrected due to cross-cancellation. The above argument on the forward-backward filtering can be mathematically summarized in the following way. Let $H(f)(=M(f) \exp [j \phi(f)])$ be the frequency response of the Butterworth filter, where $M(f)$ is the realvalued magnitude response and $\phi(f)$ is the real-valued phase response. If $S(f)$ is the signal to be filtered, $S_{f}(f)$ is the filtered signal and $S_{f-b}(f)$ is the forward-backward filtered signal, then

$$
\begin{aligned}
& S_{f}(f)=H(f) S(f)=M(f) \exp [j \phi(f)] S(f), \\
& S_{f-b}(f)=H^{*}(f) S_{f}(f)=H^{*}(f) H(f) S(f) \\
& \quad=M(f) \exp [-j \phi(f)] M(f) \exp [j \phi(f)] S(f) \\
& =M^{2}(f) S(f)
\end{aligned}
$$

where ${ }^{\prime * 1}$ denotes the complex conjugate. Since the gain responses of the forward and backward filters are identical, the signal outside the pass-band of the Butterworth filter is attenuated twice by the forward-backward filtering. The MATLAB toolbox provides a convenient function to perform the forward-backward filtering (filtfilt.m) with a given filter (in our case, the Butterworth filter). The vibration signal is then filtered with filtfilt.m and the Butterworth filter, which is shown in Fig. 4.

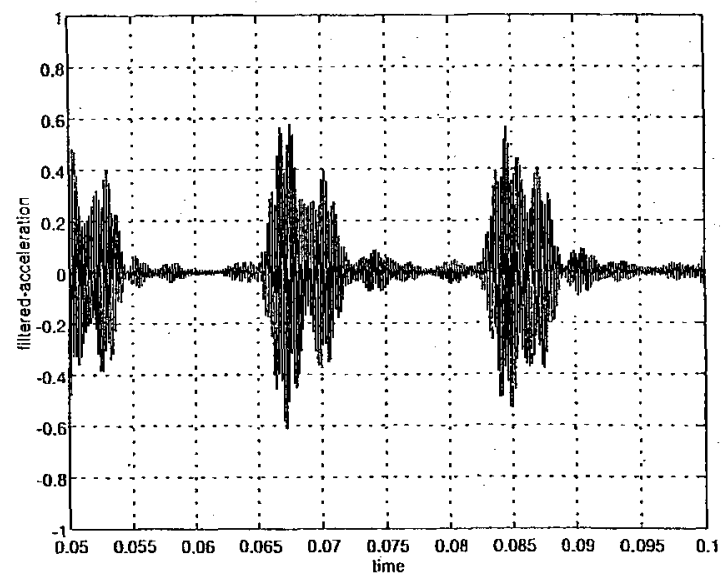

Fig. 4. Acceleration signal filtered by the $5 \mathrm{kHz}$ band-pass filter 
The filtered vibration signal is used to determine the instants in time when high amplitudes occur. Once the time instants are identified, the corresponding states of the compressor during the compression cycle can be inferred such as the position of the piston and the crank angle. Our goal is to identify the corresponding state of the compressor (identified with the crank angle) when the maximum instantaneous amplitude of the vibration signal is achieved, where it is assumed that the maximum instantaneous vibration results in maximum sound (or noise) radiation. However, the filtered signal itself cannot provide precise information on its instantaneous amplitude and the corresponding crank angle. In the following, it is shown how to determine the instantaneous amplitude via the Hilbert transform

\subsection{Estimation of Instantaneous Amplitude via Hilbert Transform}

The Hilbert transform can help to pinpoint the crank angle corresponding to maximum vibration (Simon \& Tomlinson, 1984). The Hilbert transform (or equivalently Hilbert filter) of $x(t)$ is defined as follows (Bracewell, 1986):

$$
X_{H i} \stackrel{\operatorname{def}}{=} \frac{1}{\pi} \int_{-\infty}^{\infty} \frac{x(\tau)}{\tau-t} d \tau
$$

In the frequency domain, the Hilbert transform of $x(t)$ is given by $j \operatorname{sgn}(f) X(f)$, where $\operatorname{sgn}(f)$ is 1 for positive $f$ and -1 for negative $f$, and $X(f)$ is the Fourier transform of $x(t)$ (Bracewell, 1986). Hence the Hilbert transformation is equivalent to a curious kind of filtering, in which the amplitudes of spectral components are left unchanged, but their phases are altered by $\pi / 2$ radians, positively or negatively according to the sign of $f$.

Now it is shown how the Hilbert transform can be used to obtain the instantaneous amplitude of a signal. Consider a real signal $x(t)$, which may be associated with a complex function $\hat{x}(t)=x(t)-j X_{H i}(t)$, called the analytic signal of $x(t)$. It is interesting to note that the instantaneous amplitude of a signal can be defined via the analytic signal. For example, let $x(t)=\cos \left(2 \pi f_{c} t\right)$. Then, $\hat{x}(t)=\cos \left(2 \pi f_{c} t\right)+j \sin \left(2 \pi f_{c} t\right)=\exp \left(2 \pi f_{c} t\right)$. The instantaneous amplitude of $\cos \left(2 \pi f_{c} t\right)$ is $|\hat{x}(t)|=1$. By generalizing this result, the magnitude of the analytic signal in the time domain is defined as the instantaneous amplitude, or envelope of $x(t)$ (Bracewell, 1986). Fig. 5 shows the instantaneous amplitude of the acceleration signal (IAA) at the location "A". With the IAA available, it remains to map the time instant of the maximum vibration signal into the corresponding state of the compressor (crank position). Although seemingly straightforward, it is not easy to map the specific time instant into the state of the compressor since the crank position cannot be readily revealed without internal instrumentation. However, the internal instrumentation inevitably alters the structure of the compressor, which must be avoided in order for the results of noise source identification to be valid for an undisturbed compressor. This apparent impasse is resolved through judicious selection of a secondary signal that bridges between two compressors: one with internal instrumentation and the other without internal instrumentation.

The pressure pulse signal of a compressor at the discharge line (connected to the top shell) maintains an invariant relation to the crank angle, which makes it possible to obtain the crank angle at a certain instant from the pressure signal. The relation between the discharge pulse and the crank angle depends only upon the rotational speed of the motor. The rotational speed of the motor is fairly constant around $58.5 \mathrm{~Hz}$. With this information, the following approach is proposed to measuring the vibration signal vs. crank angle without internal instrumentation and consequently without altering the compressor structure. First, a bolted-shell ${ }^{2}$ compressor is instrumented to measure the vibration signal, the pressure pulse at the discharge line, and the crank position via magnetic pick-up. Second, the vibration signal and the pressure pulse at the discharge line are measured in a compressor instrumented only externally (typically called a welded-shell compressor). Then, the pressure pulse from the bolted-shell compressor is shifted in time to match the pressure pulse from the welded-shell compressor. The delay required to match the two pressure pulses is then used to shift the magnetic pick-up from the bolted-

${ }^{2}$ The internally instrumented compressor is normally bolted after instrumentation is installed. 
shell compressor, which correspondingly provides information on the crank angle for the welded-shell compressor. In this way, the vibration signals and pressure pulses in the welded-shell compressor can be analyzed with respect to its own crank angle information. It is worth noting that the discharge cavity in the bolted-shell compressor is kept identical to that of the welded-shell compressor. Otherwise, the discharge pulse waveform would be distorted due to the different delays, which makes it impossible to compare the data obtained from the two compressors. Fig. 5 and Fig. 6 show the similarity between the pressure pulses from the bolted-shell and welded-shell compressors, which further supports our claim. Equipped with this information, the following conclusion can be drawn: the IAA over one cycle indicates that the high levels of IAA occur mostly at around $220^{\circ}$ and $300^{\circ}$.

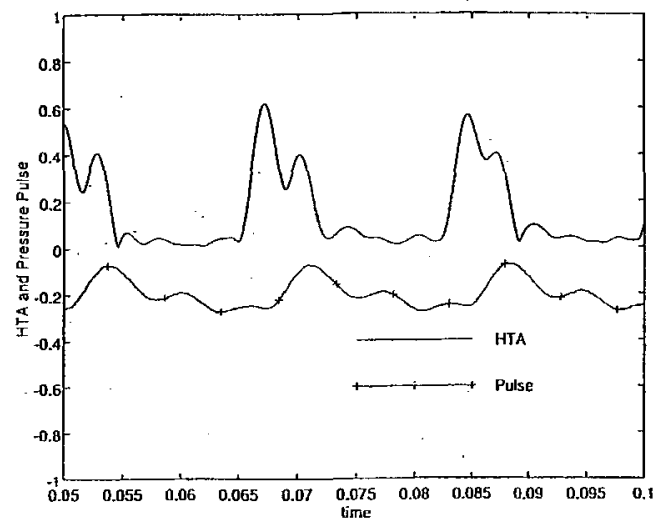

Fig. 5. Instantaneous amplitude of the acceleration signal at the location "A" (" HTA") and pressure pulse at the discharge line ("pulse") in a welded-shell rotary compressor

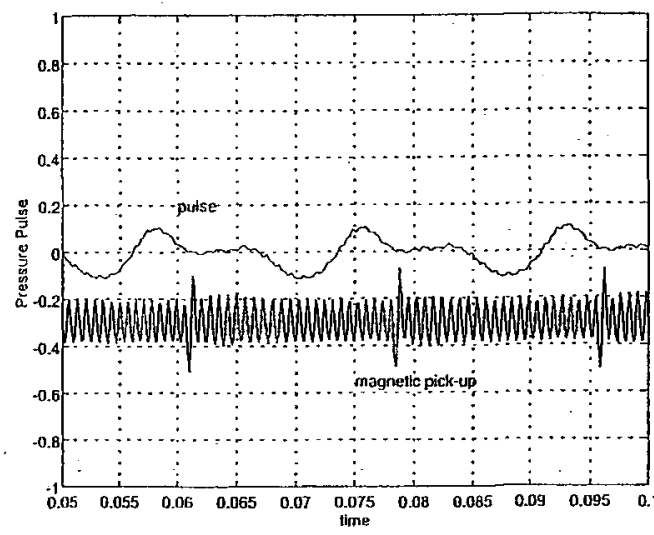

Fig. 6. Pressure pulse and magnetic pick-up in the bolted-shell rotary compressor

\section{IDENTIFICATION OF NOISE SOURCES VIA INTERNAL MEASUREMENT AND MODAL ANALYSIS}

Two noise generating mechanisms are identified from the information on the crank positions where the high levels of IAA occur: 1) over-pressurization in the cylinder chamber, and 2) structural resonance of the cylinder. The former is related to the crank angle of $220^{\circ}$ and the latter, $300^{\circ}$. The direct measurement of the cylinder pressure and the modal analysis serve as two essential vehicles in pinpointing true sources.

\subsection{Over-pressurization in the Cylinder Chamber}

The discharge valve in a rotary compressor is an automatic type valve, the name of which is coined from the fact that the pressure difference between the cylinder and the discharge plenum governs the fundamental valve opening/closing operations. Opening/closing operations of ordinary mechanical valves are mechanically synchronized with other parts of the whole system, e.g., via cams. The valve starts to open when the cylinder pressure reaches the discharge plenum pressure inside the discharge muffler. Both the cylinder pressure and the crank angle are measured in the bolted-shell compressor, noting that the cylinder pressure fluctuation is invariant to the compressor type, whether it is welded or bolted. The experimental results show that the discharge valve starts to open at a crank angle of $200^{\circ}$ when the cylinder pressure reaches the discharge plenum pressure, 311 psig, as shown in Fig. 7. The cylinder pressure continues to increase even after the discharge valve is open due mainly to the compressibility of the refrigerant. In other words, it takes a while for the cylinder pressure to reach 
the discharge plenum pressure because the refrigerant in the cylinder is compressed while the valve is open. As a result, the maximum pressure occurs at around $220^{\circ}$.

Based on the above argument and supporting experimental data, the over-pressurization is conjectured to be the candidate cause of the high level of IAA at $220^{\circ}$. In order to verify such a claim, the compressor is tested at a different discharge plenum pressure without altering the other condition: 250 psig instead of 311 psig. The experimental results show that 1) the discharge valve opens earlier than in the previous measurement, 2) the overpressurization magnitude is decreased, and 3 ) the crank angle where the cylinder pressure achieves its maximum remains unchanged at $220^{\circ}$. In Fig. 7, it is observed that the first hump at $220^{\circ}$ is reduced to a great extent due to the discharge pressure change. This validates our claim that the over-pressurization in the compression chamber is the source of the high level of IAA at $220^{\circ}$.

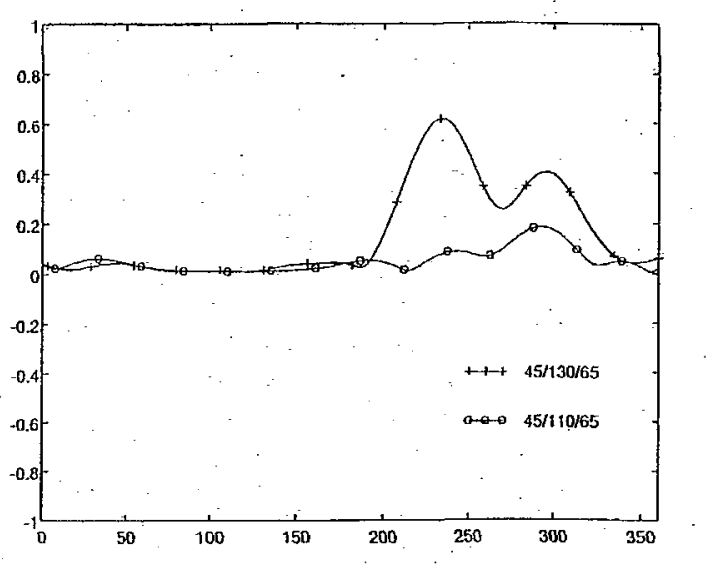

Fig. 7. Comparison of IAAs for two different discharge pressure conditions: (1) 311 psig and (2) 250 psig

\subsection{Structural Resonance of the Cylinder via Modal Analysis}

Modal analysis is the process of determining the modal parameters (natural frequencies, damping factors and modal vectors) of a linear, time-invariant system. The modal parameters can be determined via both experimentation and finite element analysis. The modal test via finite element analysis is first conducted to obtain the natural frequencies of the cylinder and the corresponding mode shapes. The results show that one of the natural frequencies is $5 \mathrm{kHz}$, which falls within our frequency range of interest. The corresponding mode shape is then computed for further analysis. The mode shape implies that the portion of the cylinder at the crank angle of $300^{\circ}$ is the most active part of the cylinder at $5 \mathrm{kHz}$. It is thus conjectured that the high vibration level at $300^{\circ}$ is caused by structural resonance. Careful examination of the compressor geometry reveals that the structural weakness (notch at the cylinder) exists at a crank angle $300^{\circ}$, which physically explains why the portion of the cylinder at $300^{\circ}$ is the most active part of the cylinder. The theoretical modal analysis is further validated experimentally via the frequency response function (FRF) measurement. The goal of the FRF test is to experimentally verify the natural frequency estimates obtained via finite element analysis (FEA). The FRF measurement is the most commonly used approach to the estimation of modal parameters (Halvosen and Brown, 1977; Goyder, 1980). The FRF is the complex ratio of the Fourier transform of output response to the Fourier transform of input excitation as a function of frequency for a single input and a single output system. The FRFs are measured using excitation at a single point (the location " $\mathrm{A}$ "). The experimental results verify the existence of the $5 \mathrm{kHz}$ natural frequency in the cylinder, which is consistent with the result of the FEA. PATRAN is used for the pre- and post-processor and ANSYS for the analysis tool (PDA Engineering, 1993; Swanson Analysis System Inc., 1992). 


\section{CONCLUDING REMARKS}

A synergetic approach is proposed for vibration-induced noise source and transmission path identification. First, the sound intensity map of a rotary compressor reveals the active region of acoustic radiation. The vibration signal is measured near the active region of the acoustic radiation. The coherence test indicates that the mechanical vibration is the main cause of the acoustic noise. Then, various signal processing techniques including band-pass filtering and the Hilbert transform are used to extract the features related to noise generation from the raw vibration signals. Finally, direct measurement and modal analysis guide us to the noise sources. The findings of the noise source identification have resulted in a successful design of quieter compressors at Carrier, Inc. By virtue of its generic nature, the applicability of the synergetic approach should be unlimited even if it has been applied to a rotary compressor

\section{REFERENCES}

1. Allemang, R. J. (1984). Experimental modal analysis bibliography. In Proc. IMAC, February.

2. Beranek, L. (1971). Noise and Vibration Control. Institute of Noise Control Engineering.

3. Bracewell, R. N. (1986). The Fourier Transform and Its Applications. McGraw-Hill, New York, Second edition, revised.

4. Crocker, M. J. (1975). Reduction of Machinery Noise. Purdue University, West Lafayette, IN.

5. Ewins, D. J. (1984). Modal Testing: Theory and Practice. Research Studies Press LTD.

6. Foreman, J. (1990). Sound Analysis and Noise Control. Van Nostrand Reinhold, New York.

7. Goyder, H. (1980). Methods and application of structural modelling from measured structural frequency response data. J. Sound and Vib., vol. 68, no. 2, pp. 209--230.

8. Halvorsen, W., \& Brown, D. (1977). Impulse technique for structural frequency response testing. J. Sound and Vib., pp. 8--21, November.

9. Harris, C. (1957). Handbook of Noise Control. McGraw-Hill, 1221 Avenue of the Americas, New York, $\mathrm{N}, \mathrm{Y} .10020$.

10. MathWorks, Inc. (1992). The PRO-MATLAB User's Guide. The MathWorks, Inc.

11. PDA Engineering (1993). PATRAN User's Manual, April.

12. Simon, M., \& Tomlinson. G. (1984). Application of the hilbert transforms in the modal analysis of non-linear systems. J. Sound and Vib. vol. 96, no. 3.

13. Swạnson Analysis System Inc. (1992). ANSYS User's Manual.

14. Yerges, L. F. (1978). Sound, Noise \& Vibration Control. Van Nostrand Reinhold, second ed. 